\title{
Curved artificial compound-eyes for autonomous navigation
}

\author{
Robert Leitel $^{* a}$, Andreas Brückner ${ }^{\mathrm{a}}$, Wolfgang Buß ${ }^{\mathrm{a}}$, Stéphane Viollet ${ }^{\mathrm{b}}$, Ramon Pericet-Camara ${ }^{\mathrm{c}}$, \\ Hanspeter Mallot ${ }^{\mathrm{d}}$, and Andreas Bräuer ${ }^{\mathrm{a}}$ \\ ${ }^{a}$ Fraunhofer Institute for Applied Optics and Precision Engineering, \\ Albert-Einstein-Str. 7, 07745 Jena, Germany \\ b Aix-Marseille Université, CNRS, ISM UMR7287, 13288, Marseille CEDEX 09, France \\ ${ }^{c}$ Laboratory of Intelligent Systems, EPFL, Station 11, CH-1015 Lausanne, Switzerland \\ ${ }^{\mathrm{d}}$ Laboratory of Cognitive Neuroscience, Department of Biology, University of Tübingen, \\ Auf der Morgenstelle 28, 72076 Tübingen, Germany
}

\begin{abstract}
Natural compound-eyes consist of a large number of ommatidia that are arranged on curved surfaces and thus are able to detect signals from a wide field of view. We present an integrated artificial compound-eye sensor system with enhanced field of view of $180^{\circ} \times 60^{\circ}$ due to the introduction of curvature. The system bases on an array of adaptive logarithmic wide-dynamic-range photoreceptors for optical flow detection and compound-eye optics for increasing sensitivity and expanding the field of view. Its assembling is mainly done in planar geometry on a flexible printed circuit board. The separation into smaller ommatidia blocks by dicing enables flexibility and finally allows for mounting on curved surfaces. The signal processing electronics of the presented system is placed together with further sensors into the concavity of the photoreceptor array, and facilitates optical flow computation for navigation purposes.
\end{abstract}

Keywords: micro-optical device, optical-flow sensor, wide field of view, comparative animal models

\section{INTRODUCTION}

The compound eyes of insects and crustaceans show an extraordinarily wide range of designs [1] as an adaption to strong varying environments from sunny daylight to dim light conditions at night or deep sea regions. Their remarkable optical layout yields high sensitivity, even polarized light sensitivity, while offering significantly larger field of view and little distortion compared to vertebrate camera eyes. Inspired by the natural antetypes, a curved artificial compound eye visual sensor has been developed and recently presented [2]. With 630 ommatidia, a field of view of $180^{\circ} \times 60^{\circ}$ and a sampling angle of about $4.3^{\circ}$, the prototype possesses characteristics similar to the eye of the fruit fly Drosophila at a weight of $1.75 \mathrm{~g}$ and a volume of $4.4 \mathrm{~cm}^{3}$. Moreover, this sensor is equipped with neuromorphic autoadaptive photodetectors and thus achieves unique visual motion sensing performance (>1000 frame/s) in a 5-decade range of illuminance.

Several attempts have been previously published to realize miniature compound eyes. Amongst them are systems based on planar microlens arrays with adjusted optical axes which have been faced to conventional flat CMOS imagers [3], [4]. Here, the limit of field of view (FOV) is about $\pm 30^{\circ}$. A way out was the introduction of micro-optics on curved substrates [5], [6], [7], including the use of spherical bulk lenses [6], planar wide angle and telescopic lenses [7], [8], and micro-prism arrays [9]. Small omnidirectional cameras [10], [11], [12], [13] designed (partly catadioptric) systems for robotic applications but consume large computational resources to correct distortion, and the use of classical imagers restricts the frame rate (e.g. $80 \mathrm{fps}$ [12]). Flexible image sensors inspired by the shape of the retina of a vertebrate "camera eye" [14], [15], and curved optoelectronic cameras were recently presented [16], [17]. Song[18] achieved an overall field of view of $160^{\circ} \times 160^{\circ}$ with 180 ommatidia by bending a stretchable planar compound eye. Again, large computational resources were required to overcome the relatively coarse resolution of the eye and the non-uniform visual sampling.

However, all approaches clarify that the realization of extremely compact compound eyes with a panoramic visual field can only be met by considering not only the optics but the whole imaging system, including the sensing elements as well as the readout electronics. As an advantage, the presented approach makes use of established technologies and rigid

Micro-Optics 2014, edited by Hugo Thienpont, Jürgen Mohr, Hans Zappe,

Hirochika Nakajima, Proc. of SPIE Vol. 9130, 91300H · (c) 2014 SPIE

CCC code: $0277-786 \mathrm{X} / 14 / \$ 18 \cdot$ doi: $10.1117 / 12.2052710$ 
materials (silicon for VLSI, glass for optics) and allows for the construction of manifold and bendable multi-channel sensor shapes. Whereas the paper by Floreano et al. [2] gives a first overview of the prototype realization and the characterization of the system, the contribution from Viollet et al. [19] focusses on the photodetectors with adaptive circuits [20] (ASIC) and the fast communication interface for data readout in order to extract motion information from natural visual scenes.

Now, we go in detail into the design and fabrication of the multi-aperture optics which is used to increase the overall sensitivity, set the angular acceptance for the ommatidia, and expands the field of view along the non-curved direction to $\pm 30^{\circ}$. The micro-optics has been realized in planar wafer-level technology using photo-lithographic patterning, resist reflow, and UV molding. In addition, the assembling technology is described in more detail, namely the aligned bonding of optics onto the ASIC, its bonding onto the flexible printed circuit board, the separation into groups of ommatidia by aligned chip-saw dicing, and the bending to a pre-defined curved scaffold.

Motion processing is useful for applications including autonomous acting terrestrial, underwater, and aerospace vehicles. Moreover, such devices are suitable as novel human-machine interfaces for domestic appliances, medical instruments, hand-held equipment, and wearables.

\section{CURVED ARTIFICIAL COMPOUND-EYE CONCEPT}

The curved artificial compound eye presented in Figure 1 consists of an ommatidial patch which has been cylindrically bent and attached to a convex shaped surface of a scaffold. The ommatidial patch has 630 photodetectors grouped in 42 identical columns of 15 photodetectors and equipped with micro-optics which are placed on a flexible printed circuit board (PCB). Two rigid PCBs bearing signal processing units have been placed in the concavity space, which are responsible for the readout of the visual data and are connected to an external processing unit. Two additional inertial sensors (3-axis rate gyro and 3-axis accelerometer) were implemented to provide complementary informations to visual data. The readout is performed using a direct serial communication process that acquires data from the parallel ommatidial columns. The ommatidial data provided by the columns is serialized at a clock frequency of $1 \mathrm{MHz}$. The visual data is delivered at a maximum frame rate of about 1500 frame per second (fps), which is within the range corresponding to fast optic flow extraction.

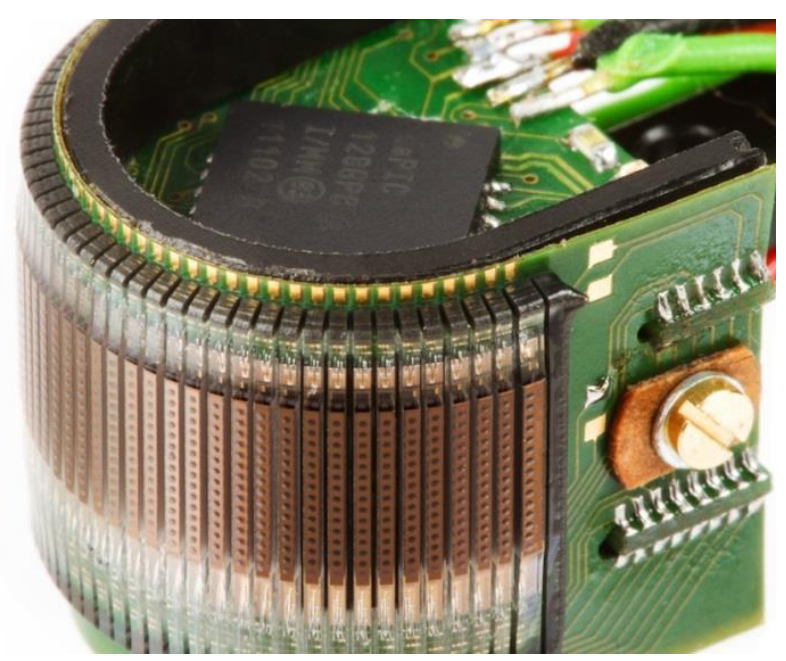

Figure 1. Left: Image of the CurvACE sensor having a diameter of $12.8 \mathrm{~mm}$, a mass of $3.5 \mathrm{~g}$, and a power consumption of $0.9 \mathrm{~W}$. The cylindrical shape was obtained from bending a rectangular array consisting of 42 columns of 15 artificial ommatidia. Right: The resulting concavity was used to house microcontrollers, rate-gyro, 3-axis accelerometer, and other electronic components.

The photodetector array has been generated on $200 \mathrm{~mm}$ multi-project wafers by $0.35 \mu$ m node CMOS technology with an optoelectronic option from XFAB giving openings with an antireflective coating at the photodiode areas. The array considers the dicing zones for column separation and thus shows 42 equal columns that consist of 15 photodetectors followed by auto-adaptive Delbrück circuits [20], a 16 channel multiplexer, a 10-bit analog-to-digital converter, a state- 
machine, and 8 electro-static discharge shielded pads for wire bonding [19]. An additional bias circuit serves to generate the polarization current required by the self-adaptive and low-pass filter circuits is integrated at the photodetector level. The width of the self-contained column's circuitry is 253 microns, and the space to its neighbors is 220 microns. This gives a column pitch of 473 microns.

The assembling makes use of planar bonding technologies for chip-level joining microoptics and ASIC as well as mounting the resulting stack onto the flexible PCB including subsequent wire bonding. By cutting a grade into the optics-ASIC-stack without touching the flexible PCB, the array of $15 \times 42$ ommatidia is separated into 42 equal columns and the whole assembly becomes flexible along one direction.

\section{MICROOPTICAL SYSTEM}

Each column contains 15 octagonal ommatidia with a diameter of $30 \mu \mathrm{m}$ width Nwell-Psubstrate photodiode. It has been shown that a photodiode with an octagonal shape has better sensitivity than a square-shaped one [21]. The photodiodes are aligned in the column with a vertical spacing of $260 \mu \mathrm{m}$.

The microoptical system is introduced in order to set the angular acceptance of each photoreceptor and to widen the field of view (FOV) in the unbent direction (along a column). The equality of acceptance angle and sampling angle results in a gapless registration of the total field of view of $180^{\circ} \times 60^{\circ}$. The sampling angle in bendable direction results from the aim to achieve a full FOV of $180^{\circ}$ and the feasible number of columns for a device. Finally, the system samples this FOV by using 42 columns with $4.3^{\circ}$ acceptance angle per ommatidium. Contrary, by applying this sampling to the rigid direction, the 15 optical channels yield a total field of view of about $60^{\circ}$. The channels are numbered from -7 to +7 identifying the central channel as 0 .

\section{Column top view}

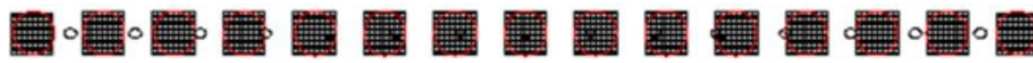

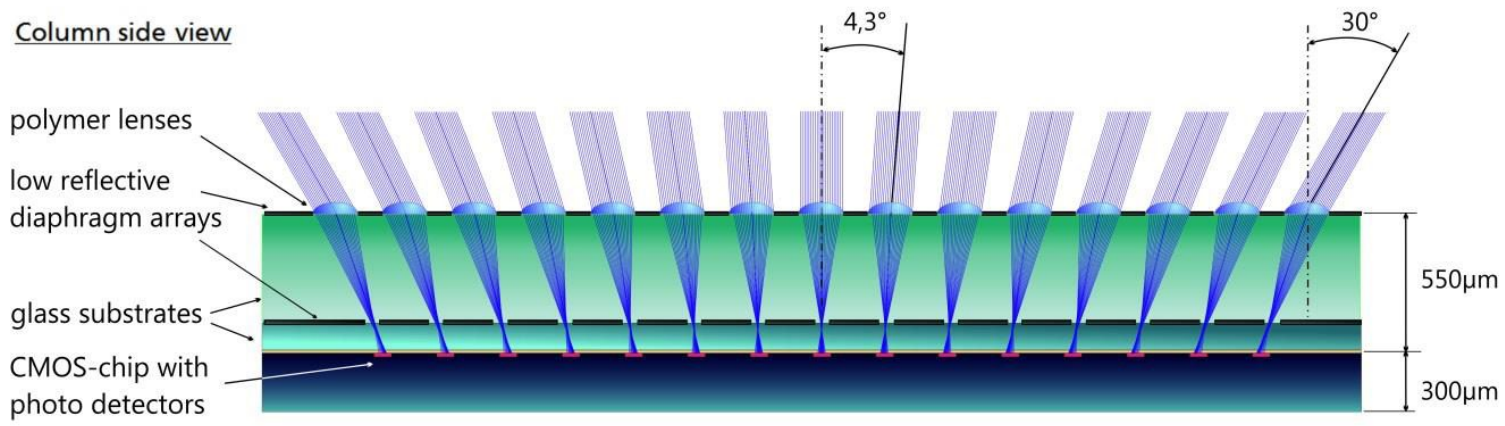

Figure 2. Schematic top and side view showing the setup of the microoptical system of a single column of the navigation sensor. Top: Red circles represent aperture opening from upper diaphragm and lens array, while small circles correspond to the lower diaphragm array. The pitch differences are used to widen the field of view.

In similarity to the natural antetype, the angular sensitivity function (ASF) of the optical channels should generate a normal (Gaussian) profile which can be technologically realized by a deliberate blurring of the lenslets - that means the lenslets do not focus at the photoreceptor plane. The registration of the slopes is favorable in combination with the Delbrück circuit for proper optics flow determination.

Using the optical raytracer software ZEMAX ${ }^{\circledR}$, a thin compound eye system has been specified with a high sensitivity (F/\# 2.4). Figure 2 shows the top and side views of a single column. The foci are not at the photoreceptor plane and cause the well-defined blurring for optics flow detection. It spans the extended FOV along a column due to the use of a microlens array with toroidal-shaped lenslets and a deviant pitch compared to the photoreceptors [22]. The pitch difference between microlens $(290 \mu \mathrm{m})$ and photoreceptor arrays $(260 \mu \mathrm{m})$ lead to off-axis paths. The variation of radii of curvature and sag height of the lenslets within the array is useful for the correction of aberration such as distortion, 
astigmatism, and field curvature, cp. Figure 3, top. The center lenslet (= channel 0 ) has a diameter of $172 \mu \mathrm{m}$ and a radius of curvature of $181 \mu \mathrm{m}$.

The simulated ASF, Figure 3 bottom, reveals a normalized (Gaussian) profile for all channels ( 0 to \pm 7 ) in sagittal cut (along the bent direction), whereas in tangential cut (along a column) a significant deviation of the slope occurs. The profile becomes asymmetric with increasing angle of incidence (or channel number) and the peak shifts by $0.8^{\circ}$, see exemplarily Figure 4 top right for the $3^{\text {rd }}$ off-axis channel. Nevertheless, the FWHM for angular acceptance, stays nearly constant $4.38^{\circ}$ (normal incidence, channel 0$)$ and $4.28^{\circ}\left(30^{\circ}\right.$ AOI, channel 7) and the slope is still evaluable for optics flow determination.

Further assumptions have been taken into account for the optics design. A diaphragm array is placed subsequent to the microlens array for aperture setting. Its openings show increased ellipticity from the center to the outer off-axis channels. A second diaphragm array is introduced close to the focal plane which is inside the glass stack. It is placed between two glass substrates of standard thicknesses of $400 \mathrm{~nm}$ and $150 \mathrm{~nm}$ which will be bonded by transparent adhesion layer. The second diaphragm array is introduced in order to prevent crosstalk between the channels, which could otherwise occur when light from a microlens will hit an image sensor pixel of an adjacent ommatidium. Moreover, the material has a low reflectivity which suppresses ghost-images from in-channel reflections.
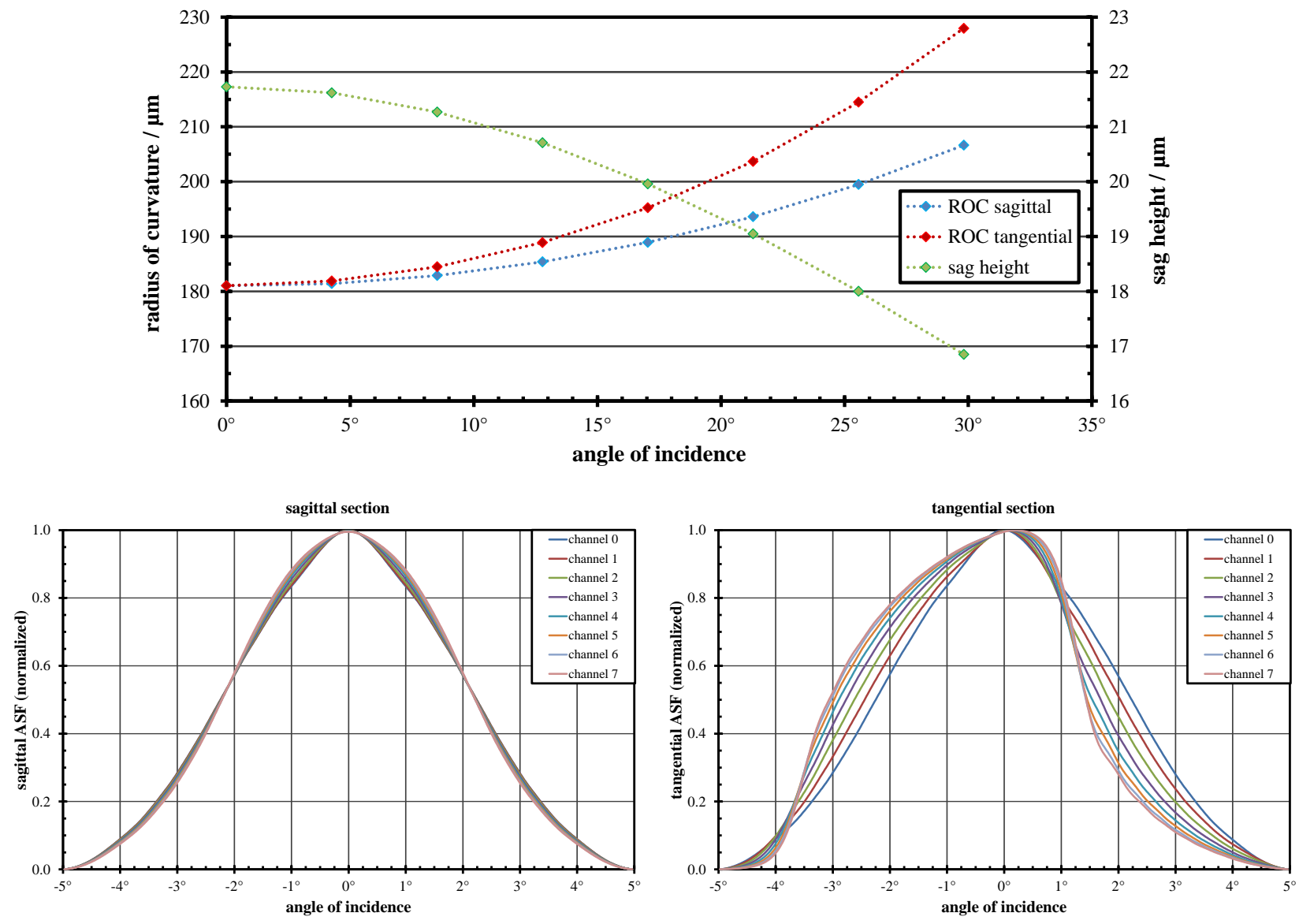

Figure 3. Top: Variation of radii of curvature and the sag height for the toroidal-shaped microlenses of a column from central channel $\left(=0,0^{\circ} \mathrm{AOI}\right)$ to channel $7\left(30^{\circ} \mathrm{AOI}\right)$; Bottom: Simulated angular sensitivity function shows the Gaussianlike behavior for sagittal direction (along the bent direction, normal incidence) and in tangential direction (along the column, off-axis incidence).

Optical crosstalk simulations have been carried out by using non-sequential ray tracing in back propagation mode, see Figure 4 (left). A Lambertian source is assumed at the image plane, whereas a detector is postulated in object space giving the relative intensity and the position of ghost images. It has been determined that off-axis ghosts may reach $0.31 \%$ relative intensity at maximum (channel $7-\mathrm{AOI} 30^{\circ}$ ). Contrary, in-channel ghosts from reflections at diaphragm 
edges may reach up to $3.7 \%$ relative intensity, Figure 4 bottom right. Fortunately, the latter do not hit active photoreceptor area.

The multi-channel imaging principle allows for a flat fabrication technology in wafer scale. The microoptics has been fabricated on 4 inch $(100 \mathrm{~mm})$ substrates of display glass (Schott Adv. Materials, D263T), Figure 5 left. Microlens masters have been generated by reflow of patterned photoresist and replicated by molding using highly transparent UVcurable organic-inorganic hybrid material (MRT GmbH, Ormocomp). The stamp material consists of transparent PDMS (Sylgard 184). Layers, which act as diaphragm arrays, are composed of lithographically patterned and wet-etched lowreflective black chromium. Because of the required alignment between the diaphragm arrays and the microlens array, a mask aligner device (Süss MicroTec, MA6) has been applied for patterning and molding. Finally, the optical stack has a total thickness of about 570 microns including the sag heights of the microlenses.
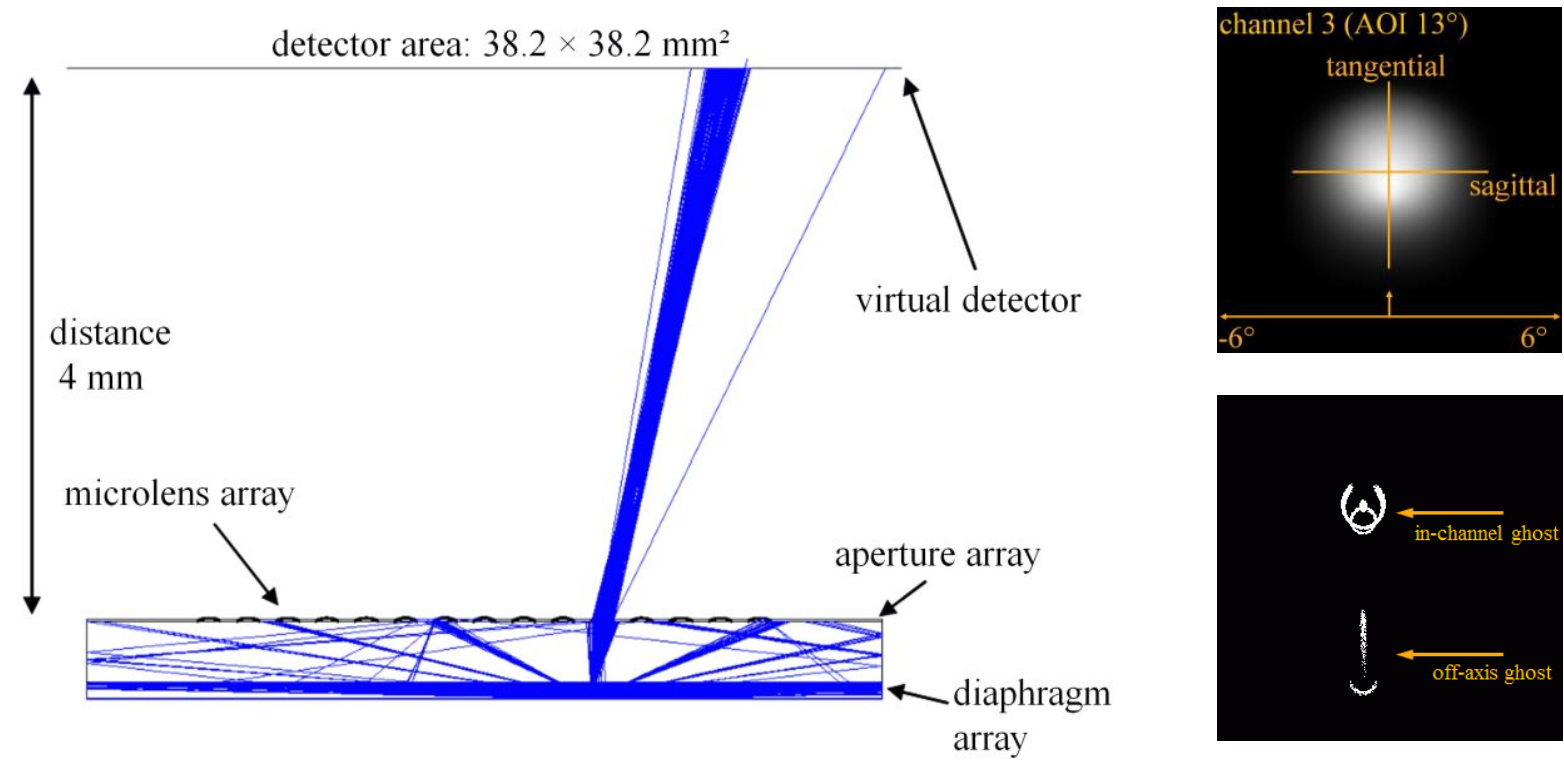

Figure 4. Left: Optical crosstalk simulation using raytracing in back propagation mode exemplarily for channel 3, and Right top: intensity distribution for at the photoreceptor plane for channel 3. Right bottom: Visualization of ghost images that may appear for channel 3 detecting intensities $I>0.01 \% I_{\max }$.

\section{ASSEMBLY AND PACKAGING}

The custom designed CMOS Opto-ASICs have been produced on multi-project wafers, and thus a wafer-level bonding of multi-aperture microoptics and sensors was not an option. Consequently, the optics has been bonded chip-wise by the help of active alignment techniques. Different assembling concepts were evaluated for prototype fabrication: Flip chip bonding would have involved the application of through silicon vias, which has been dropped as a matter of costs and available space at the sensor. Finally, wire bonding has been chosen due to the comparatively simple stack assembly of optics, ASIC, and PCB. Even more, wire bonding of dies in multi-chip modules is state of the art on flexible PCBs.

Initially, the required components have been prepared for stack mounting by separating the CMOS-ASIC and microoptics chips from 8 inch and 4 inch wafers, respectively. Additionally, the sensor chips got trenching grooves at their backside at the positions of the later dicing lanes. These trenches of $100 \mu \mathrm{m}$ depth and $150 \mu \mathrm{m}$ width make it possible to cut in a safe distance of the underlying printed circuit board (PCB), Figure 5 right. The flexible PCBs were fabricated by fine line technology with gold plated bond pads. The placement tolerance of the stack onto the PCB is $\pm 20 \mu \mathrm{m}$ due to a solder stop window which acts an alignment aid. 

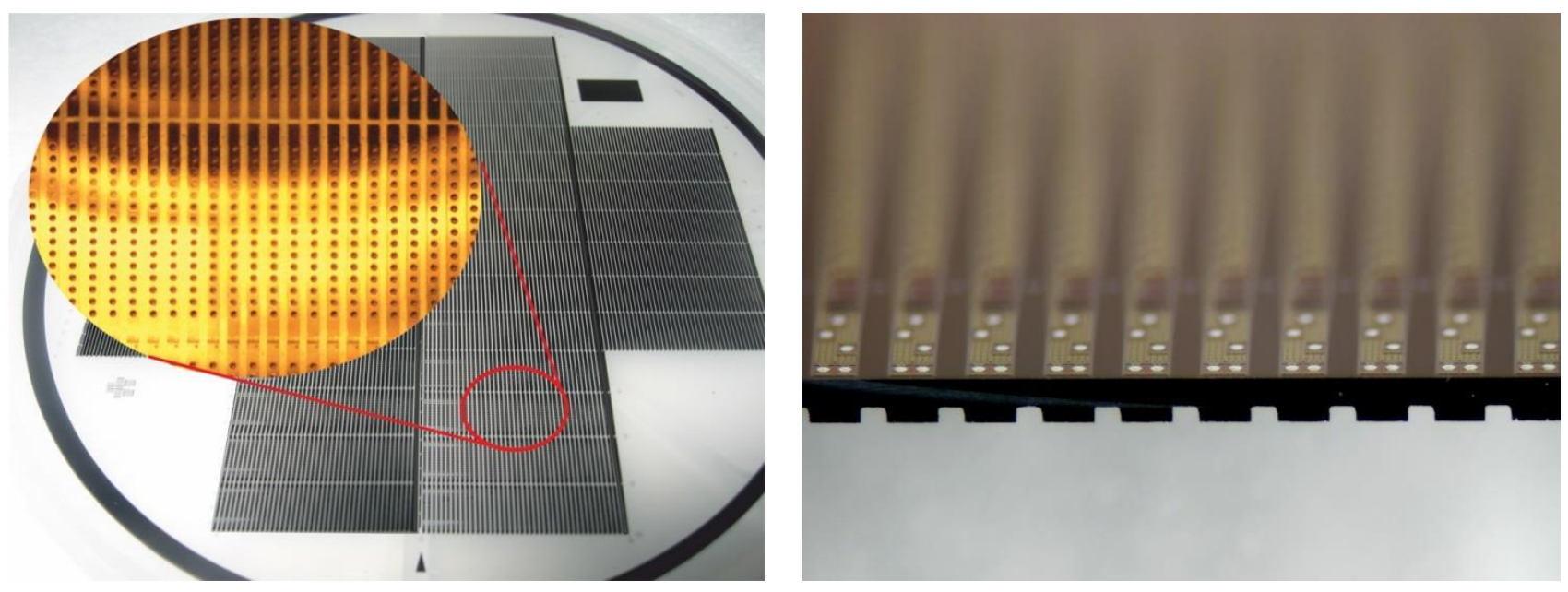

Figure 5. Left: glass wafer carrying 44 multi-channel optics chips $(42 \times 15$ channels). Right: Detailed view on separated and backside trenched CMOS-ASIC die. The grooves are at the position of the later dicing lanes which have been considered in the sensor layout.

The adhesive bonding of multi-aperture optics and CMOS ASIC was done by a FINEPLACER ${ }^{\circledR}$ lambda device using active alignment and an adapted vacuum gripper for the optics, Figure 6 left [23]. The associated alignment marks are located on the sensor's top metal layer and the optics' lower diaphragm layer. The applied transparent UV-curable adhesive (EPO-TEK ${ }^{\circledR}$ OG 146-178) exhibits high mechanical strength and heat resistance beyond $150^{\circ} \mathrm{C}$ that are appropriate for loads during column separation (shear forces) and encapsulation (heat), respectively. The accuracy of positioning is $\pm 1.5 \mu \mathrm{m}$.

The mounting of this stack onto the flexible PCB was done passive with jig aid. Again, the Fineplacer ${ }^{\circledR}$ technology would be an option, but an adapted stage was used instead which allows a faster bonding process at an adequate lower level of precision by the help of a mechanical guiding pin. The applied two-component epoxy (EPO-TEK 353ND) glue hardens at $80^{\circ} \mathrm{C}$.
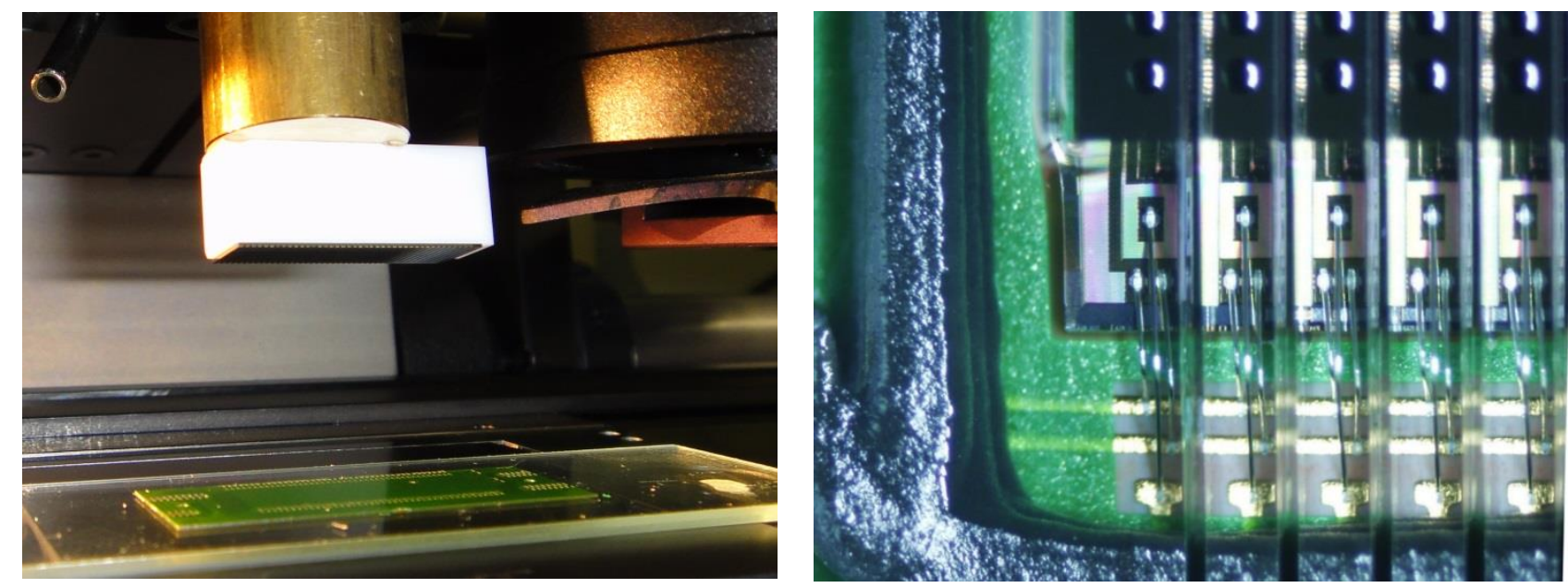

Figure 6. Left: Aligned bonding of a microoptics-sensor stack (vacuum clamped on an adapted Macor ${ }^{\circledR}$ gripper) onto a flexible PCB using a Fineplacer ${ }^{\circledR}$ device. Right: Detailed view of a corner of the CurvACE sensor after assembly. Three wires per column end have been placed and encapsulated with glob top material prior to dicing without damaging the flexible PCB with its routings.

The analog-digital circuitry and the readout require for 8 electrical interconnections per column, that group in 3 and 5 at the opposite endings and therefore lead to a total number of 336 wires. The final encapsulation step covers the bonding wires with a glob-top material, but does not spill over the optics. A previously placed polymer frame on the PCB encloses the area for casting. It is not necessary to use a transparent glob-top material, but it is valuable for the diagnostics of the wire bonds during the current prototype generation. 

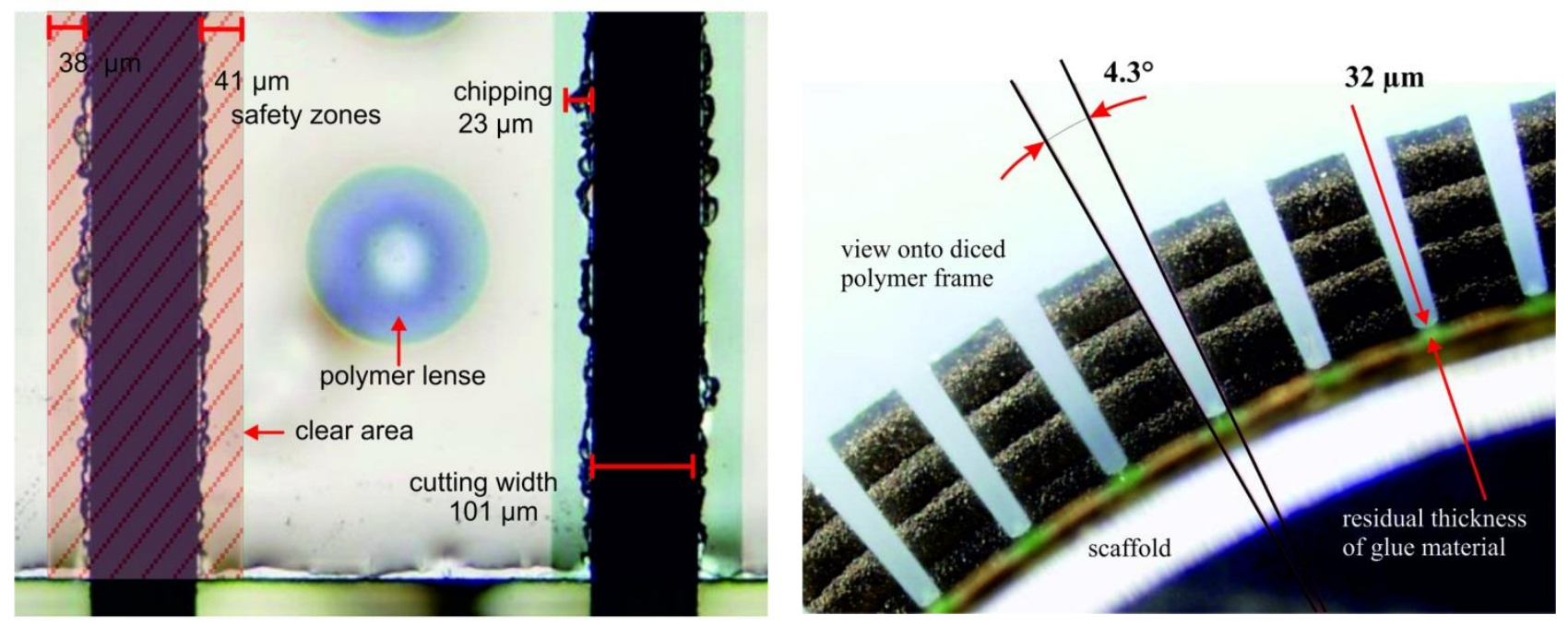

Figure 7. Left: Top-view of microoptical surface showing chipping defects, which are inside the considered safety zones, after column separation from dicing. Right: The sensor patch has been bent to the pre-defined curvature of a scaffold. The inter-channel (= interommatidial) angle is $4.3^{\circ}$. The view is from topside and shows the black polymer frame for the encapsulation material.

Precision cut-off by grinding has been applied to separate the sensor array into 42 equal columns of 15 photodetectors, see Figure 6 (right). Currently, the sawing technology is the most promising way for efficient cutting the stack of glass, silicon, and polymer of about $870 \mu \mathrm{m}$ thickness. Synthetic-resin-compound diamond saw blades of $100 \mu \mathrm{m}$ width were employed. The design of the ASIC and the microoptics considers adequate wide clear areas as dicing lines and safety zones account for chipping, cp. Figure 7 (left). The trenches at the backside ensured that the PCB was not touched by the sawing blade and stays intact. Nevertheless, residual glue in the gap between sensor and PCB causes stiffening and impede the bending. Consequently, an optimized glue thickness is inevitable to prevent uncontrolled breaks and irregular bending. Moreover, it is important to bend it exactly along its designated direction. Any torsion may cause a breaking of the columns. Two screws fix the bent PCB to the scaffold of pre-defined curvature.

\section{CURVACE CHARACTERIZATION}

The angular sensitivity has been determined for each of the 630 pixels of the CurvACE sensor. Figure 8 (right) represents exemplarily the sagittal ASFs in the azimuthal plane of the device. For measuring, the device was placed on a set of linear and rotary stages for exact orientation of the optical channel with respect to the light source, while a stepper motor drives the rotary axis in order to scan the angle of incidence in a resolution of $0.1^{\circ}$, see Figure 8 (left). The pinhole light source with a modulated intensity of $20 \mathrm{~Hz}$ (due to the Delbrïck photoreceptors) was placed in a distance of $2.8 \mathrm{~m}$.

Most ASFs display the expected Gaussian profile with respect to the light incidence angle and thus validate the microoptical design and precise alignment with respect to the photoreceptors. The experimental acceptance angles and interchannel angles were derived from the measured ASFs. The acceptance angle is defined as the full width at half maximum of its Gaussian-like ASF. The horizontal and vertical inter-channel angles were assessed from the angular position of the peak of the ASFs of two adjacent channels. The measured acceptance angles yielded an average of $4.2^{\circ} \pm 0.3^{\circ}$ (SD) for both horizontal and vertical directions. The vertical interommatidial angles resulted in an average of $4.3^{\circ} \pm 0.2^{\circ}$ (SD) and the horizontal ones ranged from $4.2^{\circ} \pm 0.8^{\circ}(\mathrm{SD})$ in the central row to $3.7^{\circ} \pm 0.7^{\circ}$ in the top and bottom rows. The close match between the experimentally measured acceptance angles and interommatidial angles validates both the ray-tracing design and fabrication process, while indicating that the CurvACE prototype performs an adequate sampling of its entire $180^{\circ} \times 60^{\circ} \mathrm{FOV}$. The observed spread in the values of the horizontal interommatidial angles can be ascribed to the manual process we used to mechanically fix the ommatidia array on to its semi-cylindrical substrate. 
The neuromorphic adaption circuit of each channel operates independently of its neighbors. The mean steady state and transient responses of the adaptive photoreceptors in response to light step increments and decrements have been verified for four different light levels while achieving high sensitivity ( 800mV/decade) [2]. At each adaptation level, the output response of the auto-adaptive photoreceptor to light steps yields an S-shaped curve in a semi-log plot.
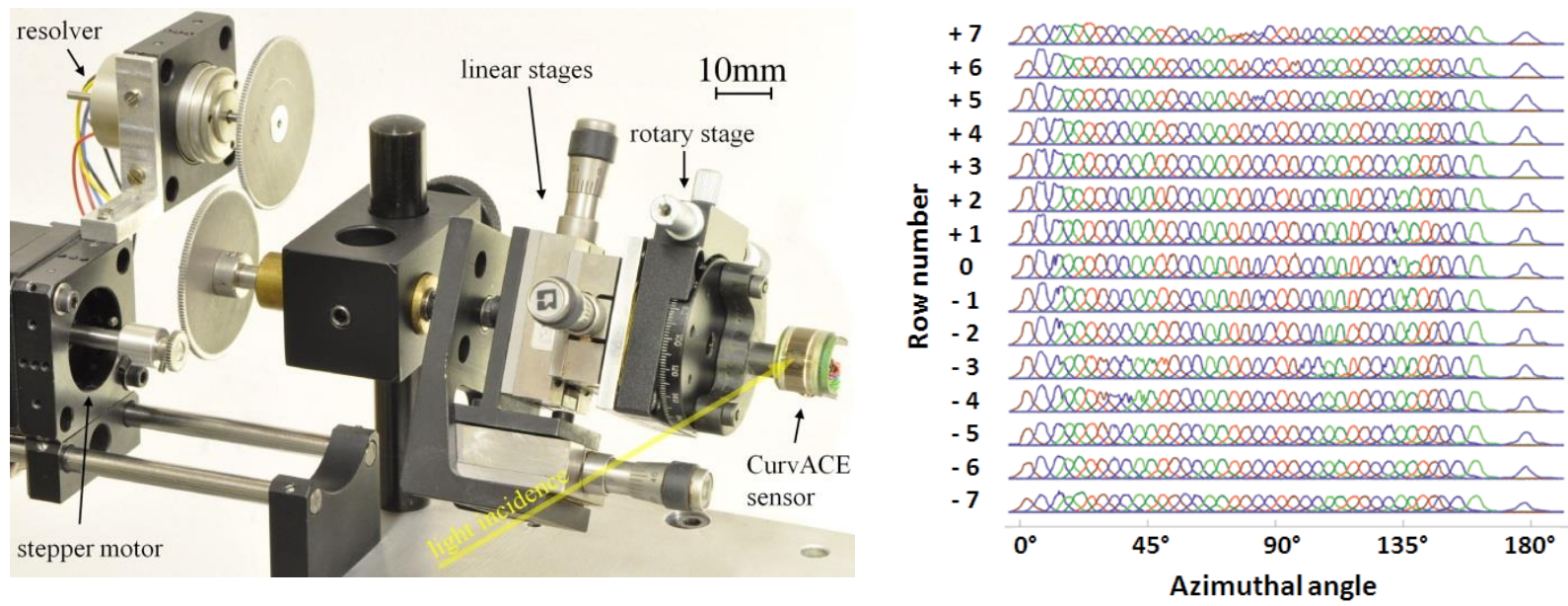

Figure 8. Left: Setup for the measurement of the ASF of CurvACE ommatidia, configured for characterizing the sagittal ASF of a row of ommatidia (azimuthal plane). The resolver measures the current angular position with a resolution of $0.08^{\circ}$. Right: Sagittal ASF for all 630 optical channels of a CurvACE demonstrator. Unfortunately, the circuitry of the $41^{\text {st }}$ column broke and the respective ASFs could not be measured in each row.

Intentionally, CurvACE has been developed as a motion-sensing device [2], but it is possible to image the moving environment. Figure 9 (top) shows the recordings when CurvACE was placed inside a rotating circular arena (diameter $1.05 \mathrm{~m}$ ) lined with a strip of paper on which the CurvACE logo was printed. To collect the full frames, the microcontrollers were connected via SPI bus to a bridge-module acting as a gateway to the USB bus of a computer.

The angular speed was $125 \%$ s, and the images were acquired at a rate of $25 \mathrm{fps}$. Despite the relatively coarse resolution, the CurvACE sensor was able to produce images of a sufficiently good quality for the pattern (part of the CURVACE logo) to be recognizable. This experiment was repeated with several levels of background illuminance ranging from 0.5 lux to 1500 lux in order to test the ability of the CurvACE sensor to compensate for changes in the illuminance. Due to the auto-adaptive Delbrück pixel, no significant differences were observed between the images obtained.

The motion detecting capability of CurvACE has been demonstrated in [2] as well. Figure 9, bottom shows exemplarily the optics flow fields for yaw and pitch rotation. The optic flow has been calculated by the non-iterative gradient-based multiframe method derived by Lucas and Kanade [24]. In order to improve the gradient calculation a bias in the response of the photoreceptors was removed. The size of the integration window for the optic flow determination is $5 \times 5$ channels and the gradient computation covers a $3 \times 3$ field. To avoid special cases for calculation at the border of the sensor, the optics flow field establishes from only $9 \times 35$ elements.

\section{SUMMARY}

The prototype of a compact cylindrical-shaped artificial compound eye has been presented which is suitable for optics flow based navigation. The well-designed equality of the acceptance angles and inter-channel angles lead to wide, gapless, and distortion-free field of view of $180^{\circ} \times 60^{\circ}$. The sampling angle of the 630 optical channels is $4.3^{\circ}$. It is straightforward to achieve $360^{\circ}$ panorama view by combining two CurvACE devices or two microoptics-CMOS stacks onto an extended flexible PCB.

The optical navigation sensor benefits from the use of auto-adaptive Delbrück photoreceptor circuits which lead to high sensitivity for fast changing contrasts while adapting (the amplification logarithmically) to slow variations of background illumination over four decades [2]. Another advantage compared to standard CMOS camera sensors, is the high sampling rate which allows for readout up to $1950 \mathrm{fps}$ [19]. 

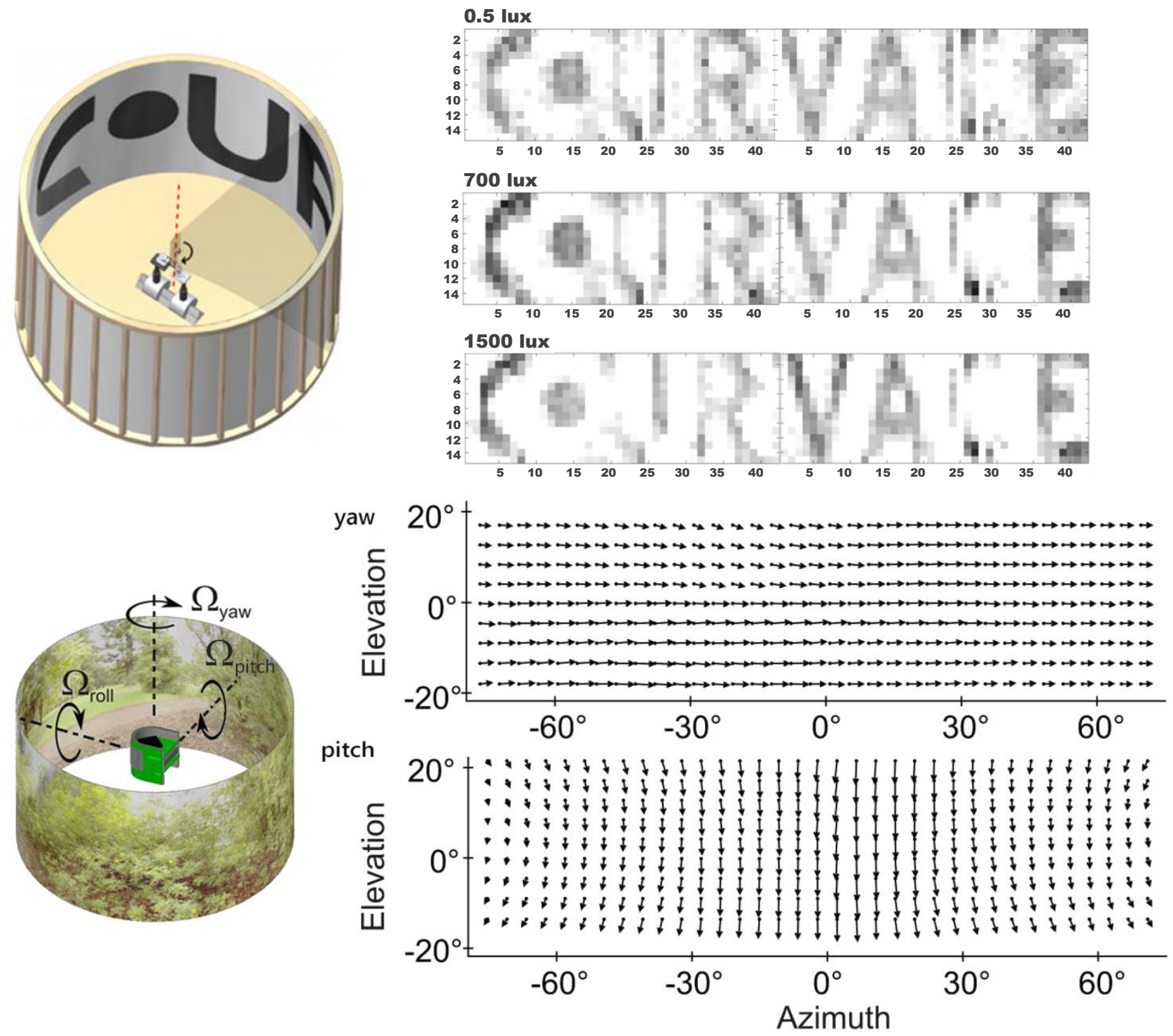

Figure 9. Top: The CurvACE device placed in a circular arena lined with the CurvACE logo. The supporting stage rotated around the vertical axis (red dotted line) at an angular speed of $125 \%$ s by means of a stepper motor. The images were acquired by CurvACE under three different lighting conditions: 0.5 lux, 700 lux and 1500 lux [19]. Bottom: CurvACE placed inside a circular arena lined with a natural print. CurvACE extracts optical flow fields, which are shown in Mercator projection exemplary for yaw and for pitch rotation at $22 \%$ [2].

The versatile approach bases on rigid materials (silicon and glass) and planar assembling technology which enables high alignment accuracy for established state-of-the-art fabrication processes [23]. They could be set-up in large scale, i.e. micro-optics fabrication, wire bonding, and precision grinding cut-off for column separation. For the shown prototype, the unidirectional bendability was used to mount the patch onto a semi-cylindrical scaffold of defined radius of curvature of $6.35 \mathrm{~mm}$ to achieve the above stated specifications. Its concavity houses the microcontrollers for generating clock and synchronization signals as well as for data readout and setting-up regions of interests and thus leads to a very compact package.

This concept can be adapted to a wide range of shapes, photoreceptor layouts, or optical resolution. Optical-flow extracting sensors will find their application in autonomous acting vehicles and robots. In addition, CurvACE will offer unique opportunities to suggest new explanations for animals' outstanding performances [25] in tasks such as navigation, obstacle avoidance, or chasing, and thus help to foster the development of visual algorithms for novel human-machine interfaces. Future improvements in the design of the compound eye may result in devices with even smaller packages, induced by progress i.e. in CMOS fabrication, processing optical materials, or precision grinding cut-off. 


\section{ACKNOWLEDGEMENTS}

The project CURVACE was financially supported by the Future and Emerging Technologies (FET) program within the Seventh Framework Program for Research of the European Commission, under FET-Open grant number 237940. This work also received financial support from the German Federal Ministry of Education and Research (BMBF). The authors gratefully acknowledge the collaboration with the CURVACE consortium from EPFL Lausanne, Aix-Marseille Université, and University of Tübingen, responsible for ASIC layout generation and CurvACE characterization, sensor integration and readout architecture, software simulation and realization, respectively, as well as the help from Felix Kraze and Andreas Mann during CurvACE assembly.

\section{REFERENCES}

[1] Land, M. F. and Nilsson, D.-E., [Animal eyes], Oxford University Press, (2012).

[2] Floreano, D., Pericet-Camara, R., Viollet, S., Ruffier, F., Brückner, A., Leitel, R., Buss, W., Menouni, M., Expert, F., Juston, R., Dobrzynski, M. K., L’Eplattenier, G., Recktenwald, F., Mallot, H. A. and Franceschini, N., "Miniature curved artificial compound eyes", Proceedings of the National Academy of Sciences 110, 9267-9272 (2013).

[3] Duparré, J. W. and Wippermann, F. C., "Micro-optical artificial compound eyes", Bioinspiration \& Biomimetics 1, R1-16 (2006).

[4] Duparré, J., Wippermann, F., Dannberg, P. and Bräuer, A., “Artificial compound eye zoom camera”, Bioinspiration \& Biomimetics 3, 046008 (2008).

[5] Jeong, K.-H., Kim, J. and Lee, L. P., "Biologically Inspired Artificial Compound Eyes”, Science 312, 557-561 (2006).

[6] Radtke, D., Duparré, J., Zeitner, U. D. and Tünnermann, A., "Laser lithographic fabrication and characterization of a spherical artificial compound eye", Optics Express 15, 3067-3077 (2007).

[7] Qu, P., Chen, F., Liu, H., Yang, Q., Lu, J., Si, J., Wang, Y. and Hou, X., “A simple route to fabricate artificial compound eye structures", Optics Express 20, 5775-5782 (2012).

[8] Kinoshita, H., Hoshino, K., Matsumoto, K. and Shimoyama, I., "Thin compound eye camera with a zooming function by reflective optics", Proc. 18th IEEE International Conference on Micro Electro Mechanical Systems MEMS, 235-238 (2005).

[9] Li, L. and Yi, A. Y., "Development of a 3D artificial compound eye", Optics Express 18, 18125-18137 (2010).

[10] Ferrat, P., Gimkiewicz, C., Neukom, S., Zha, Y., Brenzikofer, A. and Baechler, T., "Ultra-miniature omnidirectional camera for an autonomous flying micro-robot", Proc. SPIE 7000, 70000M-70000M-10 (2008).

[11] Humbert, J., Murray, R. and Dickinson, M., "A control-oriented analysis of bio-inspired visuomotor convergence", Proc. 44th IEEE Conf. Decis. Control, 245-250 (2005).

[12] Stürzl, W., Boeddeker, N., Dittmar, L. and Egelhaaf, M., "Mimicking honeybee eyes with a $280^{\circ}$ field of view catadioptric imaging system", Bioinspiration \& Biomimetics 5, 036002 (2010).

[13] Dong, F., Ieng, S.-H., Savatier, X., Etienne-Cummings, R. and Benosman, R., "Plenoptic cameras in real-time robotics", The International Journal of Robotics Research 32, 206-217 (2013).

[14] Dinyari, R., Rim, S.-B., Huang, K., Catrysse, P. B. and Peumans, P., "Curving monolithic silicon for nonplanar focal plane array applications", Applied Physics Letters 92, 091114 -091114-3 (2008).

[15] Xu, X., Davanco, M., Qi, X. and Forrest, S. R., "Direct transfer patterning on three dimensionally deformed surfaces at micrometer resolutions and its application to hemispherical focal plane detector arrays", Organic Electronics 9, 1122 - 1127 (2008). 
[16] Ko, H. C., Stoykovich, M. P., Song, J., Malyarchuk, V., Choi, W. M., Yu, C.-J., Geddes III, J. B., Xiao, J., Wang, S., Huang, Y. and Rogers, J. A., "A hemispherical electronic eye camera based on compressible silicon optoelectronics", Nature 454, 748-753 (2008).

[17] Jung, I., Xiao, J., Malyarchuk, V., Lu, C., Li, M., Liu, Z., Yoon, J., Huang, Y. and Rogers, J. A., "Dynamically tunable hemispherical electronic eye camera system with adjustable zoom capability", Proceedings of the National Academy of Sciences, (2011).

[18] Song, Y. M., Xie, Y., Malyarchuk, V., Xiao, J., Jung, I., Choi, K.-J., Liu, Z., Park, H., Lu, C., Kim, R.-H., Li, R., Crozier, K. B., Huang, Y. and Rogers, J. A., "Digital cameras with designs inspired by the arthropod eye", Nature 497, 95-99 (2013).

[19] Viollet, S., Godiot, S.,Leitel, R., Buß, W., Breugnon, P., Menouni, M., Juston, R., Expert, F., Colonnier, F., L'Eplattenier, G., Brückner, A., Kraze, F., Mallot, H., Franceschini, N., Pericet-Camara, R., Ruffier F. and Floreano D., "Hardware architecture and cutting-edge assembly process of a tiny curved compound eye" submitted to Sensors Actuators A.

[20] Delbruck, T. and Mead, C., "Adaptive photoreceptor with wide dynamic range, circuits and systems", ISCAS '94 IEEE International Symposium 4, 339 -342 (1994).

[21] Dubois, J., Ginhac, D., Paindavoine, M. and Heyrman, B., “A 10000 fps CMOS sensor with massively parallel image processing", IEEE Journal of Solid-State Circuits 43, 706-717 (2008).

[22] Duparré, J. Wippermann, F., Dannberg, P. and Reimann, A., "Chirped arrays of refractive ellipsoidal microlenses for aberration correction under oblique incidence", Optics Express 13, 10539-10551 (2005).

[23] Buß, W., Kraze, F., Brückner, A., Leitel, R., Mann, A., Bräuer, A., "Assembly of high-aspect ratio optoelectronic sensor arrays on flexible substrates", European Microelectronics Packaging Conference 2013, TuP1 (2013).

[24] Lucas, B.D. and Kanade, T., "An iterative image registration technique with an application to stereo vision", Proceedings of the 7th International Joint Conference on Artificial Intelligence (IJCAI), 674-679 (1981).

[25] Webb B., "What does robotics offer animal behaviour?", Animal Behaviour, Centre for Cognitive and Computational Neuroscience 60, 545-558 (2000). 